\title{
INCONSISTENT LIPID PROFILES EXHIBITED AMONG THE DIABETIC ASIAN INDIANS OF INDIA AND TRINIDAD-A COMPARATIVE STUDY
}

\author{
B. SHIVANANDA NAYAK' ${ }^{1}$, GEETHA BHAKTHA ${ }^{2}$ \\ ${ }^{1}$ The University of the West Indies, Faculty of Medical Sciences, Department of Preclinical Sciences, Trinidad and Tobago, ${ }^{2}$ Research \\ Scientist-II, Multidisciplinary Research Unit, Shimoga Institute of Medical Sciences, Shivamogga, Karnataka, India \\ Email:Shiv25@gmail.com
}

Received: 25 Jun 2016 Revised and Accepted: 050 oct 2016

\begin{abstract}
Objective: This was an cross-sectional observational study wherein clinical parameters were compared between the population of two countries like India and Trinidad.

Methods: One hundred and six diabetic and 100 healthy individuals from Dakshina Kannada District of India were chosen and compared with 106 Type 2 diabetic and 100 healthy individuals of Trinidad. Along with anthropometric variables and blood pressure, blood samples were collected from the subjects aged above 35years of both genders who would fit with the inclusion criteria. Total cholesterol, triglyceride, VLDL, LDL and sugar levels were estimated from the blood samples collected.
\end{abstract}

Results: Though both the study population were of similar BMI, we found considerable higher values from the baseline among the systolic pressure, total cholesterol and fasting blood glucose level in both the countries. Though the normal study population showed a small change in the mean values, most of the difference was not statistically significant. We found that Indian diabetic population had a higher risk of future complication of diabetes with significantly higher LDL $(p=0.002)$ and systolic pressure $(p=0.000)$.

Conclusion: This study shows important difference among biochemical parameters and other risk factors in the Asian phenotypic races with countries like India and Trinidad. The data also showed that Indian diabetic population are at higher risk of developing complications when compared to Trinidadians.

Keywords: Asian Indians, Diabetes mellitus, Ethnic race, Lipid profile.

(C) 2016 The Authors. Published by Innovare Academic Sciences Pvt Ltd. This is an open access article under the CC BY license (http://creativecommons.org/licenses/by/4. 0/) DOI: http://dx.doi.org/10.22159/ijpps.2016v8i12.13695

\section{INTRODUCTION}

The prevalence of type 2 diabetes mellitus (T2DM) is estimated at a projection of $4.4 \%$ increase for the year 2030 which means that 366 million persons will have T2DM [1]. Type 2 diabetes is diagnosed by a high fasting glucose level greater than $126 \mathrm{mg} / \mathrm{dL}$ and is primarily influenced by lifestyle factors such as diet and exercise. Intensive lifestyle determines the degree of adiposity accumulated which can be calculated from a person's body mass index (BMI). The measure of BMI helps in maintaining a person's ideal weight [2] and it can determine the effect adiposity will have on the body as it is a useful predictor for decreasing levels of insulin and thus the incidence of T2DM.

The decrease in insulin resistance has been shown to have effects on lipid profiles as patients who develop T2DM are observed to have higher concentrations of serum cholesterol, triglyceride and C reactive protein (CRP) in addition to lower HDL concentrations [3].

Presently India is one of the leading countries for the prevalence of T2D and has now been termed "Diabetes Capital of the world" as the number of persons diagnosed with T2D have been estimated as 80 million by the year 2030 [4]. The Asian Indians have shown to be the most dominating of all the ethnic groups in India as they were observed to have a greater waist to hip circumference and waist to hip ratio [5] together with several other distinctive features apparent for diabetes in the Asian population [6]. The "Asian Indian Phenotype" has also shown abnormalities in their biochemical parameters for irregularities in lipid profiles and was predicted to be at a higher increased risk of T2DM [7] in which genetic susceptibility showed to play a crucial role [8].

The influence of ethnicity to the development of T2DM is now establishing and although all racial groups are affected, prospective studies have shown that South Asians and African-Caribbean are some of the hardest hit groups and the risk of T2D are significantly higher among Asians, Hispanics, and blacks than among whites [9].
The occurrence of diabetes among Asian Indians has shown to be the highest when compared to the migrant Asian Indians $[10,11]$

Diabetes was also revealed to be common among all the ethnic groups of Trinidad, however, was more prevalent among the Asian Indians of the country [12]. A consensus report for 2011 revealed that East Indians accounted for $35.4 \%$ of the population, thus making them the largest ethnic group among, African, Mixed, Syrian, Caucasian, Chinese and Indigenous [13]. The occurrence of diabetes is rapidly progressing in the country of Trinidad $[14,15]$ and it is of crucial importance that this chronic condition be reduced.

This study aims to compare the diabetic and non-diabetic of the Asian phenotype for the countries India and Trinidad.

\section{MATERIALS AND METHODS}

Ethical clearance for the study was obtained from the Campus ethics committee. The individual consent was obtained from each participant and later the data, and blood samples were collected. One hundred and six diabetic and 100 healthy individuals of Dakshina Kannada District, India were chosen and compared with 106 T2D and 100 healthy individuals of Trinidad. The inclusion criteria consisted of the Asian Indian phenotype only, both diabetics and non-diabetics and persons over the age of 35 . The exclusion criteria were pregnant persons, different ethnic groups other than Asian Indian, heavy smokers and alcoholic drinkers, persons having any chemical or physical trauma, and any serious complications that could have affected the analysis. Patients were informed of the study and asked to give consent under confidentiality for their participation. Each consented patient were asked if they were aware of having any unknown micro and macrovascular conditions which were also a limitation as information was dependent on the person's knowledge. The selected patients were then assessed for physical measurements of height, weight; waist and hip circumference were obtained from which BMI was calculated after. Blood pressure was 
recorded was well. Persons were asked of their ethnicity from selfidentification.

\section{Measurement of biochemical parameters}

Fasting blood glucose levels was estimated by glucose oxidaseperoxidase method. Total cholesterol was estimated by cholesteroloxidase-peroxidase end point method. Direct determination of highdensity lipoprotein cholesterol (HDL-c) was done by immune-inhibition end point method. Triglycerides were estimated using GPO-PAP method. The very low-density lipoprotein (VLDL) was calculated using the formula VLDL=TG/5. Frieldwald's formula was used to calculate lowdensity lipoprotein (LDL)-C = Total cholesterol-HDL-(Triglycerides/5).

\section{Reagents and instruments}

All the reagents were purchased from Agape commercial company and the instrument used was fully automated obtained from Agape diagnostics.

\section{Statistical analysis}

The results were analyzed using SPSS 10.0. Descriptive analysis was used to obtain average means for serum concentrations of biochemical parameters and anthropometric data. The non-diabetic and diabetics from both countries were compared using student's unpaired ' $T$ ' test. $P$ values $<0.01$ was taken as the level of significance.

\section{RESULTS}

\section{Diabetics of trinidad and India}

The Indian population showed considerably higher weight (7.15\%), higher LDL (9.95 \%) and lower HDL (-26.82 \%) as shown in table1.0 and fig. 1.0 with significantly lower age while the Trinidadian population displayed significantly higher fasting glucose. Both diabetic populations displayed high systolic pressure and similar cholesterol and BMI.

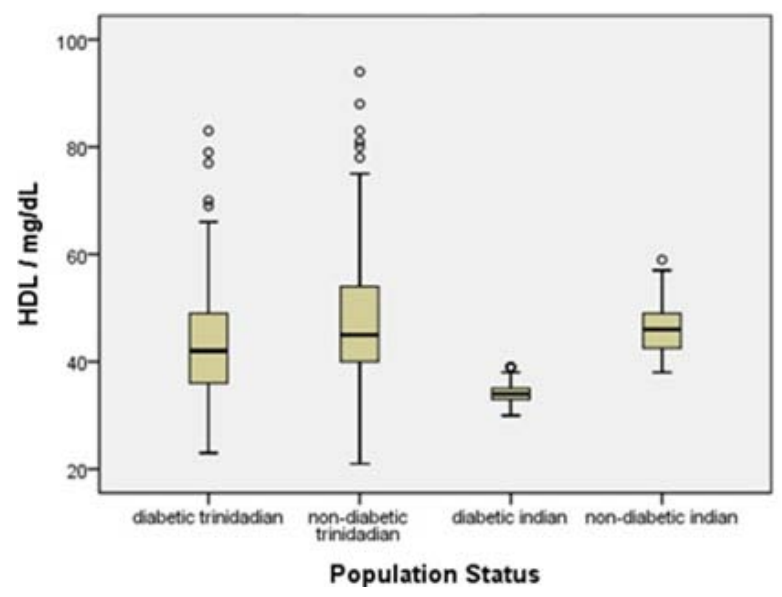

Fig. 1: HDL status of Trinidadian and Indian population

\section{Non-diabetics of trinidad and India}

The non-diabetics of the Indian population cholesterol (-5.63\%), triglyceride (-8.34\%) and LDL (-6.05\%) when compared to the nondiabetics of Trinidad population even though both non-diabetic populations had similar age, weight, and BMI.

Table 1: Demographic and anthropometric data for Trinidad and India

\begin{tabular}{|c|c|c|c|c|c|c|}
\hline \multirow[t]{2}{*}{ Variable } & \multicolumn{2}{|c|}{ Diabetic $(n=106)$} & \multirow[t]{2}{*}{$p=0.05$} & \multicolumn{2}{|c|}{ Non-diabetic $(n=100)$} & \multirow[t]{2}{*}{$\mathrm{p}=\mathbf{0 . 0 5}$} \\
\hline & Trinidad & India & & Trinidad & India & \\
\hline Age & $56.10 \pm 8.092$ & $47.68 \pm 8.140$ & $0.000^{*}$ & $46.62 \pm 14.613$ & $46.53 \pm 8.898$ & 0.950 \\
\hline Weight & $69.47 \pm 15.737$ & $74.82 \pm 9.718$ & $0.003^{*}$ & $68.11 \pm 16.524$ & $68.33 \pm 7.979$ & 0.950 \\
\hline Glucose & $156.83 \pm 75.441$ & $137.23 \pm 36.093$ & $0.002^{*}$ & $90.91 \pm 30.044$ & $74.34 \pm 12.443$ & $0.010^{*}$ \\
\hline Cholesterol & $213.53 \pm 115.150$ & $204.48 \pm 34.078$ & 0.314 & $204.75 \pm 43.020$ & $193.82 \pm 20.560$ & 0.237 \\
\hline Triglyceride & $167.46 \pm 105.103$ & $141.87 \pm 43.972$ & $0.013^{*}$ & $144.30 \pm 90.854$ & $133.19 \pm 30.390$ & 0.292 \\
\hline $\mathrm{HDL}$ & $43.54 \pm 11.383$ & $34.33 \pm 1.860$ & $0.000^{*}$ & $47.30 \pm 14.704$ & $46.25 \pm 4.462$ & 0.131 \\
\hline LDL & $127.67 \pm 40.173$ & $141.78 \pm 31.775$ & $0.002^{*}$ & $128.25 \pm 37.844$ & $120.93 \pm 20.920$ & 0.124 \\
\hline Systolic pressure & $136.30 \pm 18.971$ & $125.11 \pm 11.209$ & $0.000^{*}$ & $129.94 \pm 19.823$ & $125.00 \pm 9.036$ & $0.025^{*}$ \\
\hline Diastolic pressure & $81.39 \pm 9.310$ & $100.51 \pm 7.665$ & $0.000^{*}$ & $79.99 \pm 11.208$ & $90.52 \pm 7.694$ & $0.000^{*}$ \\
\hline BMI & $26.88 \pm 5.623$ & $27.03 \pm 4.627$ & 0.827 & $25.92 \pm 6.279$ & $26.69 \pm 3.337$ & 0.283 \\
\hline
\end{tabular}

*Significant at $95 \%$ confidence level, mean \pm SD,

\section{Overall trinidad and India}

Both the diabetic and non-diabetic groups from India displayed significantly higher diastolic pressures when compared to the both groups in Trinidad (fig. 2.0).

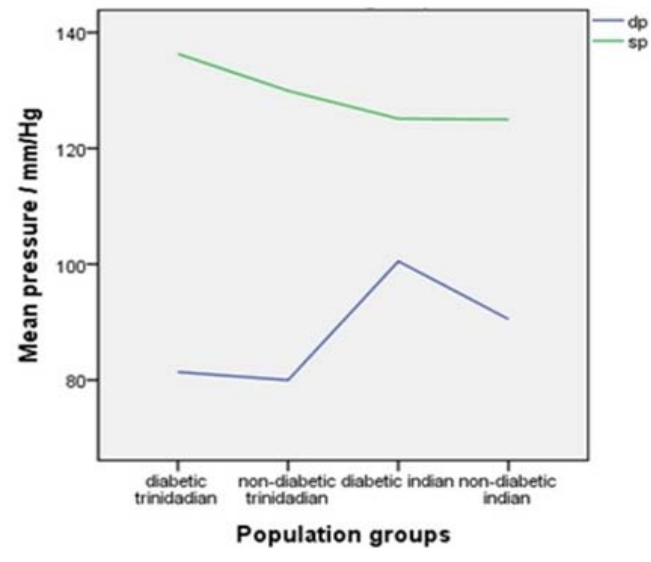

Fig. 2: Blood pressure status of all groups

\section{Gender groups in Trinidad and India}

The Trinidad groups consisted of more females than male while conversely the India groups had more males than females (fig. 3.0). The India population exhibited a greater number of males with lower HDL (fig. 4.0).

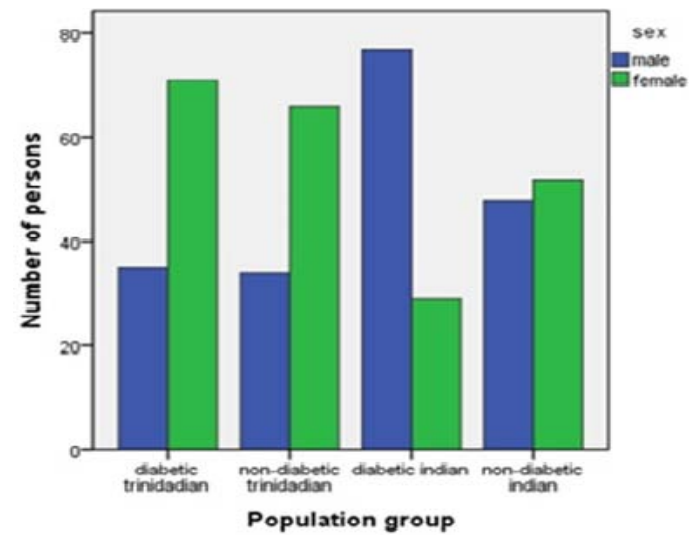

Fig. 3: Gender distribution 


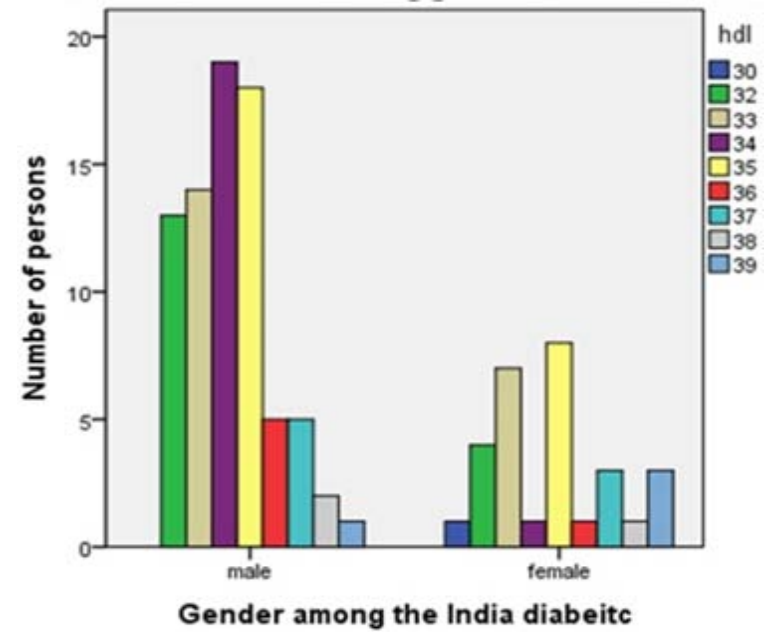

Fig. 4: Distribution of HDL among Indian subjects

\section{DISCUSSION}

The Asian Indian phenotype has been known to have profound characteristic features when compared to other ethnicities thus making them particularly susceptible to chronic conditions. This genetic makeup can be found worldwide, however, dependant on location, environment, lifestyle and other factors that they have been observed to differ significantly in anthropometric and biochemical parameters. The body composition of Asian Indians is that they have abnormal fat distribution to with prevalent high truncal obesity which makes them more prone to insulin resistance and dyslipidemia.

When a comparative study was conducted among the Asian Indians of Trinidad and Asian Indians of India, the diabetic population from India was observed to have significantly higher weight with much lower HDL values. This is standard as HDL aids in fat transportation away from blood vessels to the liver for processing, and therefore a person with a low HDL can result in a higher percentage of fat accumulating thus accounting for an increase in weight as previous research have evaluated this correlation $[16,17]$.

One of the most outstanding features of the Asian Indian phenotype is their unique body type that consists of lower BMI with higher truncal adiposity and body fat which is apparently known as thin-fat Indian phenotype [18]. Another comparative study done with Indian and Malaysian population showed the poor diabetic control, anthropometric and lipid profile among male was higher when compared to females [19]. The study population of India had a significantly higher male to female ratio in which the males were presented with lower HDL values thus placing the males at a greater risk for CAD which was outlined in a previous study [20]. Conversely, in Trinidad, the male population were at higher risk for $\mathrm{CAD}$ when compared to females [21]. This is another significant finding as the females are inclined to have more central adiposity when compared to the men.

A possible aetiopathogenesis for this can be explained by the genetic predisposition of the Asian Indians from their country or their environmental factors such as economic prosperity, urbanization, and decreased physical activity, high intake of refined cereal, low dietary fiber and low fruit and vegetable intake [22].

The diabetics from both populations displayed high systolic pressures above the average range thus increasing their risk for hypertension. A more unusual finding revealed that both the diabetics and non-diabetic of India displayed significantly higher diastolic pressures when compared to the diabetic and non-diabetic of the Trinidadian population. This uncommon outcome suggests that the Indians were at an increased risk for diastolic hypertension.

Overall the diabetic and non-diabetic Trinidadian displayed much higher levels of glucose which can be accounted for their lifestyle, diet, and level of exercise when compared to the Indian population which was a major limitation in this study. The Indian diabetic population showed lower HDL cholesterol and higher LDL when compared to Trinidadians. A proper assessment must be made to compare these parameters for the same ethnic group in order to help in the prevention and management of diabetes

\section{ACKNOWLEDGEMENT}

Authors would like to thank all the staff of Adarsha Multispecialty hospital of India and Regional health authorities of Trinidad. We also extend our sincere thanks to Ms. S Mohammed for helping in writing this article.

\section{Contribution details}

Both authors are responsible for designing, data analysis and writing the article.

\section{CONFLICT OF INTERESTS}

Authors declare that there is no conflict of interest.

\section{REFERENCES}

1. Shaw RAS, Zimmen PZ. Global estimates of the prevalence of diabetes for 2110 and 2030. Diabetes Res Clin Pract 2010;87:4-14.

2. Geneva W. World Health Organization. Global Health Estimates: Deaths by Cause, Age, Sex and Country 2000-2012; 2014.

3. Organization WH. Global status report on non-communicable diseases 2014 Geneva; 2012.

4. Hu FB MJ, Stampfer MJ, Colditz G, Liu S, Solomon CG, Willett WC. Diet, lifestyle, and the risk of type 2 diabetes mellitus in women. N Engl J Med 2001;345:790-7.

5. Anthony JG, Hanley PAJK, Ken Williams, Andreas Festa, Ralph B, D'AgostinoJr, Lynne E, et al. Prediction of type 2 diabetes mellitus with alternative definitions of the metabolic syndrome the insulin resistance atherosclerosis study. Am Heart Assoc 2005;112:3713-21.

6. Iris Shai P, Rui Jiang, JoAnn E Manson, Meir J Stampfer, Walter C Willett, Graham A Colditz, et al. Ethnicity, obesity, and risk of type 2 diabetes in women-A 20-year follow-up study. Diabetes Care 2006;29:1585-90.

7. Mohan SS, Deepa BS, Varghese C. Epidemiology of type 2 diabetes: Indian scenario. Indian J Med Res 2007;125:217-30.

8. Ramachandran ASC, Viswanathan V, Viswanatha MHS. The risk of non-insulin dependent diabetes mellitus conferred by obesity and central adiposity in different ethnic groups: a comparative analysis between Asian Indians, Mexican Americans and Whites. Diabetes Res Clin Pract 1997;36:121-5.

9. Ramachandran AM, Ronald Ching Wan Ma, Snehalatha C. Diabetes in Asia. Lancet 2009;375:408-18.

10. Deepa R SS, Mohan V. Abdominal obesity. Abdominal obesity, visceral fat and type 2 diabetes-"Asian Indian phenotype. In: Mohan V, Rao GHR, editors. Type 2 diabetes in South Asians: Epidemiology, risk factors and prevention. New Delhi: Jaypee Brothers Medical Publishers; 2006. p. 138-52.

11. Zimmet P. Type 2 (non-insulin-dependent) diabetes-an epidemiological overview. Diabetologia 1982;22:399-411.

12. International Diabetes Federation [Internet]; 2003.

13. Ramachandran ASC, Viswanathan V. Low risk threshold for acquired diabetogenic factors in Asian Indians. Diabetes Res Clin Pract 2004;65:185-95.

14. Blaak E. Gender differences in fat metabolism. Curr Opin Clin Nutr Metab Care 2001;4:499-502.

15. Ramachandran ASC, Kapur A, Vijay V, Mohan V, Das AK, Rao PV, et al. High prevalence of diabetes and impaired glucose tolerance in India: National Urban Diabetes Survey. Diabetologia 2001;44:1094-1.

16. Williams P, Robinson D. High-density lipoprotein and coronary risks factors in normal men. Lancet 1979;1:72-5.

17. Thompson PD, Jeffery RW, Wing RR, Wood PD. Unexpected disease in plasma high-density lipoprotein cholesterol with weight loss. Am J Clin Nutr 1979;32:2016-21.

18. Deepa R, Sandeep S, Mohan V. Abdominal obesity, visceral fat and type 2 diabetes. Asian Indian Phenotype 2006;2:138-52.

19. Ali QB, Hassan Y, Abdullah JD. Ifferences in demographics, lipid profile and other clinical characteristics among type 2 diabetic patients in the state of Penang, Malaysia according to 
gender and races. Asian J Pharm Clin Res 2011;4(Suppl 2):130-3.

20. Lee J, Heng Derrick, Chia KS, Chew SK, Tan BY, Hughes K. Risk factors and incident coronary heart disease in Chinese, Malay, and Asian Males: the Singapore cardiovascular cohort study. Int J Epidemiol 2001;30:983-8.

21. Poon-King T, Henry MV, Rampersad F. Prevalence and natural history of diabetes in Trinidad. Lancet 1968;291:155-60.
22. Unnikrishnan R, Anjana RM, Mohan V. Diabetes mellitus and its complications in India. Nat Rev Endocrinol 2016;12:357-70.

\section{How to cite this article}

- B Shivananda Nayak, Geetha Bhaktha. Inconsistent lipid profiles exhibited among the diabetic asian indians of india and trinidad-a comparative study. Int J Pharm Pharm Sci 2016;8(12):60-63. 